\title{
Study on Emergency Load Shedding Based on Frequency and Voltage Stability
}

\author{
Zhichao Zhang ${ }^{1,2}$, Zengping Wang ${ }^{1}$ and Panyu Fang ${ }^{1}$ \\ ${ }^{1}$ State Key Laboratory for Alternate Electrical Power System with Renewable Energy \\ Sources, North China Electric Power University, Beijing 102206, China; \\ ${ }^{2}$ College of Mechanical and Electronic Engineering, Shihezi University, Shihezi \\ 832003, China \\ 1tianfuchao@sohu.com, ${ }^{2}$ wangzp1103@sina.com, ${ }^{3}$ cheerup1990@163.com
}

\begin{abstract}
Under frequency load shedding is widely used under emergency situations in the power system. It significantly ensures frequency stability of power system by dropping excessive loads at certain places. In order to improve the traditional UFLS schemes, the rate of frequency change is taken into consideration when calculating the quantity of active power imbalance in the power system. The measure factors of frequency change rate are analyzed and a new function is created to calculate the active power imbalance based on frequency and voltage. Moreover, a new method which utilizes frequency and voltage sensitivity to determine the location and corresponding quantity of load shedding is proposed. Voltage stability should be maintained at the time of recovering system frequency stability. The simulation results based on IEEE39 buses system show that the new self-adaptive scheme of load shedding is more reasonable and superior.
\end{abstract}

Keywords: active power imbalance, the rate of frequency change, voltage sensitivity, UFLS (under frequency load shedding), frequency stability, voltage stability

\section{Introduction}

The current power systems usually operate on the system stability limitation. In order to ensure the integrity and security of the whole system, it has become a difficult problem to design effective protection and control strategies reasonably. In terms of UFLS strategy, the existing algorithms [1-11] can be divided into four kinds: the traditional method, the semiadapted method, the self-adaptive method and the computer-aided algorithm. The simulation calculation mainly depends on two models, namely single generator-load and multi-generator load frequency response model. The first two algorithms mostly use the single generator-load model to model and simulation and these belong to the category of static stability study, the other two realize dynamic load shedding strategy by using dynamic frequency response model and real-time electric parameters information.

When power system suffers a big disturbance, the voltage drops almost instantaneously. This is reflected in the loads' real and reactive power and resulted in decreasing the consumption of load power affected by voltage. Because the mismatch between power generation and load power consumption should become decreasing compared to the normal system bus voltage, the change value of system frequency become smaller or keep the same. This kind of phenomena prevents the system frequency drop to the UFLS setting point, the load dropped by UFLS will get less relative to the quantity required by system. Due to slow 
frequency reduction, the action time of UFLS is forced to extend and it is easier to cause power system blackout by cascading failure $[12,13]$. The major drawback of using the frequency-response model for UFLS is the fact that the load's voltage dependence is not included in the load model.

With the development of WAMS (wide area measurement system, WAMS) technology, the function such as the monitoring, evaluation and control of the whole system can be realized [14] feasibly. This paper focuses on analyzing the advantages and disadvantages of the traditional UFLS strategies, the measurement factors influencing frequency change rate are analyzed in detail. The variation function between the unbalance real power and the frequency change is derived accurately after the system gets disturbed. Furthermore, more attention is paid to the location of load shedding and the ability of system stability recovery after given part of load is dropped. A new scheme is proposed that the imbalance of power system is confirmed adaptively by using the rate of frequency change and voltage change. Then voltage sensitivity is used to determine the locations and the corresponding quantity of load shedding. The simulations including the traditional UFLS scheme and the advised new strategy were carried out in IEEE 39 buses test system to verify the feasibility and effectiveness of the advised new project. The results show that new scheme of load shedding is more reasonable and superior.

\section{System Frequency Response Analysis}

\subsection{The analysis of rotor movement swing}

When the power system suffers a disturbance, the oscillation among each generator units is existent. For this reason, the measurement frequency of local generator bus cannot provide enough information all the system. The concept of COI (centre of inertia, COI) has been widely used in electric power systems [15], single generator rotor swing balance equation as follows:

$$
\frac{2 H_{\mathrm{i}}}{f_{\mathrm{N}}} \frac{\mathrm{d} \Delta f_{i}}{\mathrm{~d} t}=\Delta P_{i}
$$

Where the $H_{i}$ is the inertia constant in $\mathrm{s}, f_{\mathrm{N}}$ the rated frequency in $\mathrm{Hz}, \Delta P_{i}$ is the system's unbalanced power in per unit. By add $\mathrm{n}$ differential generator swing equations, one obtains the following expression for the imbalance power of total load-generation:

$$
\Delta P=\sum_{i=1}^{n} \Delta P_{i}=\frac{2 \sum_{i=1}^{n} H_{i}}{f_{\mathrm{N}}} \frac{\mathrm{d} \Delta f_{\mathrm{COI}}}{\mathrm{d} t}
$$

Where $\Delta f_{\text {COI }}$ is the expression of the COI of system frequency, $\mathrm{d} \Delta f_{\mathrm{COI}} / \mathrm{dt}$ is the rate of frequency change.

$$
\Delta f_{\mathrm{COI}}=\frac{\sum_{i=1}^{n}\left(H_{i} \Delta f_{i}\right)}{H_{\mathrm{eq}}}, H_{\mathrm{eq}}=\sum_{i=1}^{n} H_{i}
$$

The moment of initial disturbances in the system, the swing equation for the equivalent system as follow:

$$
\left.\frac{\mathrm{d} \Delta f_{\mathrm{COI}}}{\mathrm{d} t}\right|_{t=0}=\frac{f_{\mathrm{N}}}{2 H_{\mathrm{eq}}} \Delta P=\frac{f_{\mathrm{N}}}{2 H_{\mathrm{eq}}}\left(\Delta P_{\mathrm{M}}-\Delta P_{\mathrm{L}}\right)
$$


$\Delta P_{\mathrm{M}}$ and $\Delta P_{\mathrm{L}}$ are separately the change of the mechanical power and the electric power in per unit. In equation (4), the relationship between $\mathrm{d} \Delta f_{\mathrm{COI}} / \mathrm{dt}$ and the electric parameters can be determined at $\mathrm{t}=0 \mathrm{~s}$. And the value is assured by the changed $\Delta \mathrm{P}$ and $H_{\text {eq. }}$.

$\Delta P$ is defined in per unit based on the system volt-ampere base, $S_{\text {eq }}$ which is said to be equal to the sum of the ratings of all the generating units in the system. $P_{\text {def }}$ is the active power deficits in MW.

$$
\Delta P=\frac{P_{\text {def }}}{\sum_{i=1}^{n} S_{i}}=\frac{P_{\text {def }}}{S_{\text {eq }}}
$$

Considering (3)-(5), $P_{\text {def }}$ in MW can be written as:

$$
P_{\text {def }}=\frac{2 H_{\text {eq }} S_{\text {eq }}}{f_{\mathrm{N}}} \frac{\mathrm{d} \Delta f_{\mathrm{COI}}}{\mathrm{d} t}
$$

Therefore, the measured value $P_{\text {def }}$ from (6) includes load changes. One of the possibilities to model the load mathematically is as follows:

$$
\left\{\begin{array}{l}
P_{L}=\sum_{i=1}^{m} P_{L 0, i}\left(U_{i} / U_{0, i}\right)^{\alpha_{i}}\left[1+K_{P}\left(\Delta f / f_{0}\right)\right] \\
Q_{L}=\sum_{i=1}^{m} Q_{L 0, i}\left(U_{i} / U_{0, i}\right)^{\beta_{i}}\left[1+K_{Q}\left(\Delta f / f_{0}\right)\right]
\end{array}\right.
$$

where $P_{\mathrm{L}}$ and $Q_{\mathrm{L}}$ represent the current values of the system loads' active and reactive powers respectively, $P_{\mathrm{L} 0, i}$ and $Q_{\mathrm{L} 0, i}$ represent the $i$ th load's initial active and reactive power just before the disturbance, $U_{i}$ is the current voltage on the $i$ th load bus, $U_{0, i}$ is the voltage of the $i$ th load bus just before the disturbance, $\mathrm{m}$ is the number of load busses in the system, $\alpha_{i}$ and $\beta_{i}$ is the factors depicting the active-power and reactivepower dependence of the $i$ th load on voltage deviations. $K_{P}$ and $K_{Q}$ is the factors of load frequency dependence.

\subsection{The measurement analysis of the rate of frequency change}

When the power system suffers disturbance, the main factors influencing $P_{\text {def }}$ [16] including: pre-disturbance mechanical power on the turbine $\mathrm{P}_{\mathrm{M} 0}$, active power load $\mathrm{P}_{\mathrm{L} 0}$, speed characteristics $R$ and available output capacity $\Delta P_{\mathrm{M}}$. After disturbance, $\Delta P_{\mathrm{L}}$ is the value caused due to the change of load bus voltage and system frequency. Overview of important variables relevant for determining the active power deficit in the system via the frequency gradient is shown in Figure 1.

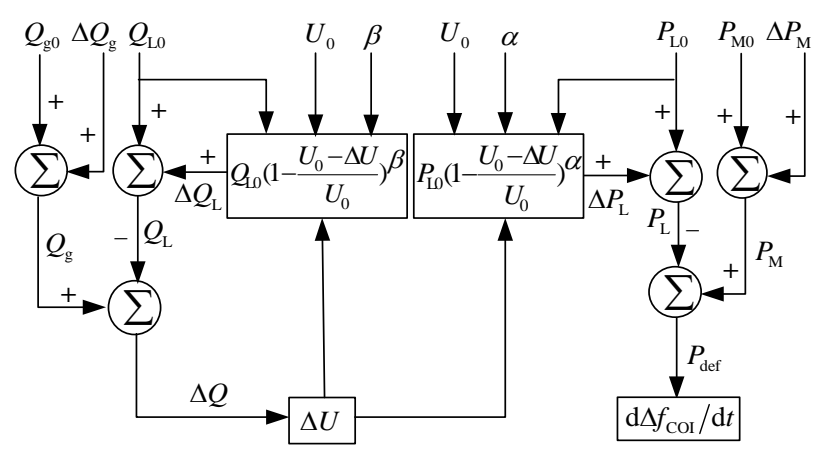

Figure 1. Viable relation determining the rate of frequency change 
After the disturbance, the speed of voltage change response is faster. The mechanical power $P_{\mathrm{M}}$ is the sum of $P_{\mathrm{M}, 0}$ and $\Delta P_{\mathrm{M}}$, neglecting the frequency dependence of the load for the present. Ignoring the internal loss of generator, the difference of $P_{\mathrm{M}}$ and $P_{\mathrm{L}}$ is $P_{\text {def }}$ which will cause the speed change of generator rotor. Frequency and its change rate are the indicator of active power unbalance, and the degree of power deficiency in the system can be reflected. Reactive power of generator is $Q_{\mathrm{g} 0}$ before disturbance, the control amount along with the voltage change is $\Delta Q_{\mathrm{g}}, Q_{\mathrm{g}}$ is the post-disturbance reactive power. $\Delta Q$ is the difference of $Q_{\mathrm{g}}$ and $Q_{\mathrm{L}}$, which will be reflected through actual voltage change $\Delta U$, the voltage change in reverse impact not only reactive load $\Delta Q_{\mathrm{L}}$ but also active load $\Delta P_{\mathrm{L}}$.

According to the analysis of Figure 1, the drawback here is not the value we seek for determining the amount of required total shedding. Namely, it is not reasonable to presume that the system will recover stability after the load shedding is completed. And the remaining load, to a great extent, reaches its initial values of active and reactive power. Neglecting other system losses, $P_{\text {def }}$ can be written as

$$
P_{\text {def }}=P_{\mathrm{M}}-P_{\mathrm{L}}
$$

Inserting the load characteristics (7) into (8), we can retrieve the value of the required total load shedding amount in MW.

$$
\begin{aligned}
P_{\mathrm{M}}-P_{\mathrm{L} 0} & =P_{\mathrm{M}}-\sum_{i=1}^{m} P_{\mathrm{L} 0, i} \\
& =P_{\text {def }}+\sum_{i=1}^{m} P_{\mathrm{L} 0, i}\left[\left(U_{i} / U_{0, i}\right)^{\alpha_{i}}-1\right]
\end{aligned}
$$

As we will show, the change in the frequency gradient due to voltage deviations is considerable, and depends also on the initial loading of the system. For this reason, consideration of the voltage deviations is of importance. Considering (6) and (9), the rate of frequency change can be written as:

$$
\begin{aligned}
\frac{\mathrm{d} \Delta f_{\mathrm{COI}}}{\mathrm{d} t} & =\frac{f_{\mathrm{N}}}{2 H_{\mathrm{eq}} S_{\mathrm{eq}}}\left[\left(P_{\mathrm{M}}-P_{\mathrm{L} 0}\right)+\sum_{i=1}^{m} P_{\mathrm{L}, i}\left[1-\left(U_{i} / U_{0, i}\right)^{\alpha_{i}}\right]\right] \\
& =\Delta f_{1}+\Delta f_{2}
\end{aligned}
$$

From (10) it is evident that $\mathrm{d} \Delta f_{\mathrm{CO}} / \mathrm{dt}$ consists of two parts, denoting as $\Delta f_{1}$ and $\Delta f_{2}$. The first part determines the rate of frequency change when the load consists of the constant active and reactive power. The second part, on the other hand, determines the contribution of the load change to $\mathrm{d} \Delta f_{\mathrm{COI}} / \mathrm{dt}$ due to the load's voltage change. As shown in figure 1 and the analysis above, if constant power model is adopted in the system, namely $\alpha=0, \beta=0$, then $\Delta f_{2}$ can be ignored. It is evident that the initial system load $P_{\mathrm{L} 0}$ and voltage change $\Delta U$ play important roles on the value of $\Delta f_{2}$. When $\Delta f_{2}$ compared to $\Delta f_{1}$ become bigger, $\Delta f_{2}$ must be considered.

\section{New Load Shedding Strategy Analysis}

This paper proposes a new adaptive load shedding algorithm based on WAMS. The main goal of the scheme is that load shedding is carried out under emergency situations, the stability of system frequency can be restored, and the stability of voltage does not be destroyed after the load shedding is implemented. This strategy has two main steps, the amplitude of system disturbance is firstly assured adaptively according to the 
disturbance situation, and then the location of load shedding and the corresponding quantity are determined according to the voltage sensitivity of load bus. So, rapid shedding of the load required can be realized, and the stability of whole system may be recovered feasibly.

\subsection{Determining the disturbance amplitude}

For an adaptive load shedding strategy, it is undoubtedly the most important to determine the deficiency of system power accurately. As for the actual power gaps between electric power and load consumption, consider the capacity of system spinning reserve $P_{\mathrm{SR}}$, the load power needing to be adjusted can are obtained by formula (9), namely, $P_{\text {shed }}$ is the total removal amount of the system required as follow:

$$
P_{\text {shed }}=\left(P_{\mathrm{SR}}+P_{\text {def }}\right)+\sum_{i=1}^{m} P_{\mathrm{L} 0, \mathrm{i}}\left[\left(U_{i} / U_{0, \mathrm{i}}\right)^{\alpha_{i}}-1\right]
$$

$P_{\text {shed }}$ is the most important parameter as for input variables of the system disturbance. If all the capacity of generation and load power can be acquired in the system, $P_{\text {shed }}$ can directly be determined by (8). Otherwise, inserting (6) into (11), the relationship between $P_{\text {shed }}$ and $\mathrm{d} \Delta f_{\mathrm{COI}} / \mathrm{dt}$ as follow:

$$
\begin{aligned}
P_{\text {shed }} & =\frac{2 H_{\mathrm{eq}} S_{\mathrm{eq}}}{f_{\mathrm{N}}} \frac{\mathrm{d} \Delta f_{\mathrm{COI}}}{\mathrm{d} t}+P_{\mathrm{SR}}+\sum_{i=1}^{m} P_{\mathrm{L} 0, i}\left[\left(\frac{U_{i}}{U_{0, i}}\right)^{\alpha_{i}}-1\right] \\
& =a \frac{\mathrm{d} f_{\mathrm{COI}}}{\mathrm{d} t}+b
\end{aligned}
$$

Where a $=\mathrm{g}_{1}\left(S_{\mathrm{eq}}, H_{\mathrm{eq}}\right), \mathrm{b}=\mathrm{g}_{2}\left(U_{i}, \alpha_{i}, P_{\mathrm{L} 0, i}, P_{\mathrm{SR}}\right)$, two functions defined is as follows:

$$
\begin{gathered}
a=\frac{2 H_{\mathrm{eq}} S_{\mathrm{eq}}}{f_{\mathrm{N}}} \\
b=\sum_{i=1}^{m} P_{\mathrm{L} 0, i}\left[\left(U_{i} / U_{0, i}\right)^{\alpha_{i}}-1\right]+P_{\mathrm{SR}}
\end{gathered}
$$

As the analysis, formula (12) is just the accurate expression of the deficiency in the power system. For different $P_{\mathrm{L} 0, \mathrm{i}}$ and $\alpha_{i}$, the actual power deficiency is always changed. The consumption of load power is connected with $P_{\mathrm{L} 0, i}$ and $\alpha_{i}$ and $U_{i}$. Because of considering the voltage variation, the time of determining $P_{\text {shed }}$ is quicker and accurate than only adopting the rate of frequency change. A certain margin is also left to calculate $P_{\text {shed }}$ more.

\subsection{Determining the location of load shedding}

After $P_{\text {shed }}$ is identified, the next need determine the location and corresponding quantity of load shedding. The change of the node voltage reflects the information whether the distribution of reactive power is balanced in the system [17].

Although the voltage amplitudes is widely used in UVLS (under voltage load shedding, UVLS) scheme [12], but the judge of the voltage collapse margin by the voltage descend is not quite accurate in general. When the system suffers a big disturbance, it is not reliable to determine the location of load shedding only according to the voltage amplitude. 
In the system, the expression for transmission equation of active power $P$ and reactive power $Q$ [16] as follows.

$$
\left\{\begin{array}{l}
P_{i}=V_{i} \sum_{j=1}^{n} V_{j}\left(G_{i j} \cos \theta_{i j}+B_{i j} \sin \theta_{i j}\right) \\
Q_{i}=V_{i} \sum_{j=1}^{n} V_{j}\left(G_{i j} \sin \theta_{i j}-B_{i j} \cos \theta_{i j}\right)
\end{array}\right.
$$

Where $V_{i}, V_{j}, G_{i j}, B_{i j}$ and $\theta_{i j}$ are bus voltage and admittance between any node $i$ and $j$, phase angle difference. With the help of Jacobi matrix, equation (15) may be expressed in the linear form:

$$
\left[\begin{array}{c}
\Delta P \\
\Delta Q
\end{array}\right]=\left[\begin{array}{ll}
\frac{\partial P}{\partial \theta} & \frac{\partial P}{\partial \mathrm{V}} \\
\frac{\partial Q}{\partial \theta} & \frac{\partial Q}{\partial \mathrm{V}}
\end{array}\right]\left[\begin{array}{c}
\Delta \theta \\
\Delta V
\end{array}\right]=\left[\begin{array}{ll}
J_{\mathrm{P} \theta} & J_{\mathrm{PV}} \\
J_{Q \theta} & J_{\mathrm{QV}}
\end{array}\right]\left[\begin{array}{c}
\Delta \theta \\
\Delta V
\end{array}\right]
$$

Where $\Delta P$ is the incremental change in bus real power, $\Delta Q$ is the incremental change in bus reactive power injection, $\Delta \theta$ is the incremental change in bus voltage angle, $\Delta V$ is the incremental change in bus voltage magnitude.

System voltage stability is affected by both $P$ and $Q$. However, at each operation point we may keep $P$ constant and evaluate voltage stability by considering the incremental relationships between $Q$ and $V$. This is analogous to the $Q-V$ curve approach. Although incremental changes in $P$ are neglected in the formulation, the effects of changes in system load or power transfer level are taken into account by studying the incremental relationship between $Q$ and $V$ at different operating conditions. Based on the above considerations, in equation (16), let $\Delta P=0$, then

$$
\begin{aligned}
\Delta Q & =\left[J_{\mathrm{QV}}-J_{\mathrm{Q} \theta} J_{\mathrm{P} \theta}^{-1} J_{\mathrm{PV}}\right] \Delta V \\
& =J_{\mathrm{R}} \Delta V
\end{aligned}
$$

Where $\mathrm{J}_{R}$ is the reduced Jacobian matrix of the system. From Equation (17), we may write:

$$
\Delta V=J_{\mathrm{R}}^{-1} \Delta Q
$$

The $V-Q$ sensitivity at a bus represents the slope of the $Q-V$ curve at the given operating point. A positive $V-Q$ is indicative of stable operation, the smaller the sensitivity, the more stable the system. As the system stability decreases, the magnitude of the sensitivity increases, becoming infinite at the stability limit. Conversely, a negative $V-Q$ is indicative of unstable operation [18]. $V-Q$ sensitivity reflects the internal relations between the system voltage and reactive power. The load bus, which voltage stability is no good, should be the point of load shedding, and the more load should be removed.

According to all bus voltage sensitivity $\left(\mathrm{d} V_{i} / \mathrm{d} Q_{i}\right)$ of the load, the load shedding position and the quantity are determined. The flow diagram of new strategy is put forward, as shown in Figure 2. The equation (19) expresses the quantity of load shedding at each load bus, $P_{\text {shed,i }}$ 


$$
P_{\text {shed }, i}=\frac{\mathrm{d} V_{i} / \mathrm{d} Q_{i}}{\sum_{i=1}^{m}\left(\mathrm{~d} V_{i} / \mathrm{d} Q_{i}\right)} P_{\text {shed }}
$$

Prepared parameters

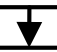

After disturbance, the control centre acquiring all bus voltage $V$ and generators frequency $f$

Acquiring the sensitivity of all system load bus $\mathrm{d} Q_{i} / \mathrm{d} V_{i}$

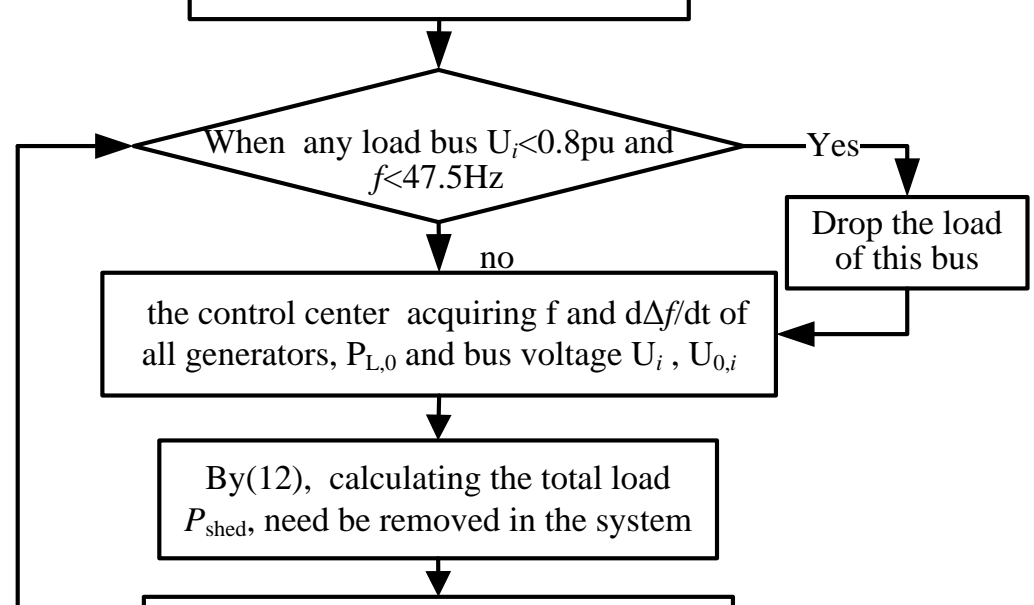

Caculating the quantity of each load bus $\mathrm{P}_{\text {shed }, i}$ by equation (19)

Determining action step and the time for load shedding

Figure 2. flow chart of load shedding scheme

Table 1. setting of traditional UFLS scheme

\begin{tabular}{cccc}
\hline Round & $\begin{array}{c}\text { Operating } \\
\text { frequency }\end{array}$ & $\begin{array}{c}\text { The proportion of } \\
\text { the removal }(\%)\end{array}$ & Export delay(s) \\
\hline basic 1 round & 49 & 8 & 0.2 \\
basic 2 round & 48.75 & 8 & 0.2 \\
basic 3 round & 48.5 & 8 & 0.2 \\
basic 4 round & 48.25 & 8 & 0.2 \\
basic 5 round & 48 & 8 & 0.2 \\
special 1 round & 48.8 & 8 & 15 \\
special 2 round & 48.6 & 8 & 15 \\
\hline
\end{tabular}




\section{The Simulation Example Analysis}

Using standard IEEE 39 nodes example in this paper, all the system power is $6150 \mathrm{MW}$ and 1409MVar in normal situation. Simulation model is established in BPA, five-order model is adopted in generator, the governor model is the type I, and excitation model is the IEEE standard DC1 model. In order to analyze conveniently, $\alpha=2$ and $\beta=2$ are adopted in load model, every generator has $10 \mathrm{MW}$ real power reserve capacity, the capacity of reactive power has a small margin.

Two kinds of perturbation are simulated. One is the example of dropping generator unit; the other is to open an important tie-line. The generator capacity of node 30 is smaller in the system, the generator of node 32 has more real and reactive power capacity, line 16-19 has the greater transmission capacity. For each of the perturbation, the system stability is observed and analyzed before and after the traditional UFLS and new load shedding strategy. In this paper, the setting of the traditional UFLS is shown in Table 1.

\subsection{The simulation analysis of tripping generator}

Tripping node 30 generator, $250 \mathrm{MW}$ real power and145MVar reactive power is removed at the same time. The change curves of load bus voltage and system frequency are shown in Figure 3. The reserve capacity of system real power is $90 \mathrm{MW}$, active power gap is $160 \mathrm{MW}$. The curve of the voltage change in load bus 7, bus 16 and the generator-load bus 31 in the system is shown in Figure 3. Bus 7 and bus 16 are just the nodes without generator, and the node 31 has both generator and load at the bus.

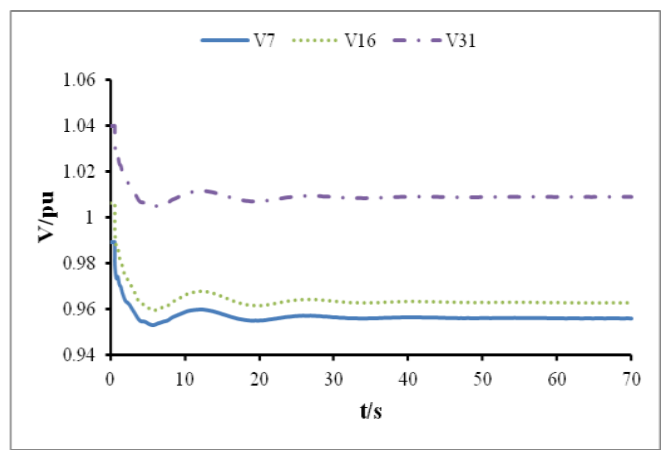

(a) Voltage change of the load bus

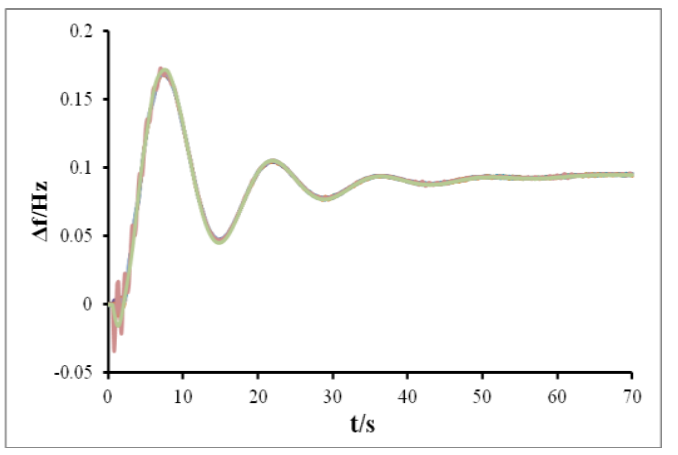

(b) The change of generator frequency

\section{Figure 3. the change curves of load bus voltages and generator frequency response}

Observing (a) of the Figure 3, after generator 30 trips, the reactive power supply of the system should get reduced. This should results in a decline of load bus voltage, but the voltages will not descend to the point where the system will lose the stability. Observing (b) of the figure 3 , the load bus voltage of the system will descend, real power load depending on the voltage become much smaller in multiples of the voltage square. This makes up the part of real power deficiency in the system, the steady frequency of the system may not fall after the disturbance, and stabilizing to a new operating point, even in the range of stable operation. In an emergency institution, the traditional UFLS strategy does not meet the action conditions, but the system has the possibility of voltage instability. 
Therefore, when calculating the actual unbalanced power in the system, the influence of the voltage dependence on load power must be considered. It is proved that the actual quantity of load shedding should be calculated using the formula (12) in the adaptive emergency control strategy.

\subsection{The analysis of tripping an important tie-line}

The key degree of the line in the system can be judged according to its influence on the system. Line 16-19 is considered as the key line in this example. It will split two generator of node 33 and 34 due to its disconnection, and the loss capacity of the system is $1140 \mathrm{MW}$, two islands are formed in the system. For the island lack of G33 and G34, spare reserve capacity is $80 \mathrm{MW}$. Big power deficiency will make the system appear a serious real power imbalance, and the system will be in an emergency state. It is urgent to adopt load shedding control strategy for preventing the system stability from further damage. The transmission power is 454MW and 454MVar before the disturbance. Under the new scheme, considering the spare capacity, the amount of load shedding should be $375 \mathrm{MW}$. The load bus voltage sensitivity and corresponding load shedding distribution are shown in Table 2.

Table 2. Voltage sensitivity and load shedding quantity of each load bus

\begin{tabular}{ccccc}
\hline Load bus number & $\mathrm{d} V_{i} / \mathrm{d} Q_{i}$ & load shedding percent $(\%)$ & $P_{\text {shed }, i}(\mathrm{MW})$ & $Q_{\text {shed }, i}(\mathrm{MV}$ ar $)$ \\
\hline bus3 & 0.0037 & 0.06 & 20.29 & 0.15 \\
bus4 & 0.0033 & 0.04 & 18.10 & 6.66 \\
bus7 & 0.0021 & 0.05 & 11.52 & 4.14 \\
bus8 & 0.002 & 0.02 & 10.97 & 3.70 \\
bus12 & 0.0026 & 1.90 & 14.26 & 88.00 \\
bus15 & 0.0077 & 0.13 & 42.23 & 20.19 \\
bus16 & 0.0091 & 0.15 & 49.90 & 4.89 \\
bus18 & 0.0056 & 0.19 & 30.71 & 5.83 \\
bus20 & 0.0019 & 0.02 & 10.42 & 1.58 \\
bus21 & 0.0063 & 0.13 & 34.55 & 14.50 \\
bus23 & 0.0034 & 0.08 & 18.65 & 6.37 \\
bus24 & 0.0082 & 0.15 & 44.97 & 13.43 \\
bus25 & 0.0015 & 0.04 & 8.23 & 1.73 \\
bus26 & 0.0032 & 0.13 & 17.55 & 2.15 \\
bus27 & 0.0049 & 0.10 & 26.87 & 7.17 \\
bus28 & 0.0016 & 0.04 & 8.77 & 1.18 \\
bus29 & 0.0011 & 0.02 & 6.03 & 0.57 \\
Bus39 & 0.0013 & 0.03 & 6.76 & 1.53 \\
\hline
\end{tabular}

4.2.1 The analysis of traditional UFLS strategy: The action level of load shedding strategy is in $49 \mathrm{~Hz}$, a total of $492 \mathrm{MW}$ active loads are dropped, at the same time, including 112.72MVar reactive loads. The change curves of load bus voltage and system frequency are shown in Figure 4. By using the traditional UFLS scheme, about 117MW more loads are dropped. System Frequency and bus voltage can restore to the safe and stable range, the deviation of system frequency is about $1 \mathrm{~Hz}$, and the time for system stability recovery is about 60 seconds. 


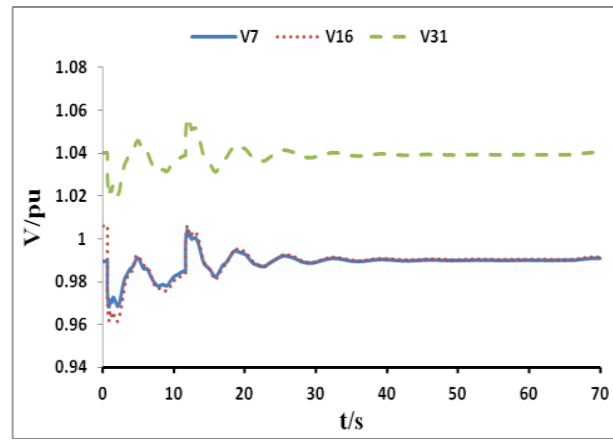

(a) Voltage change of the load bus

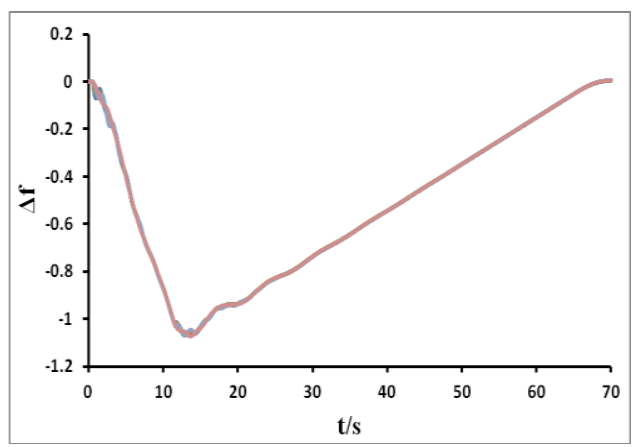

(b) The change of generator frequency

Figure 4. the change curves of load bus voltage and generator frequency using traditional UFLS scheme

4.2.2 The analysis of new load shedding strategy: New scheme considers system data's collection, processing and action time delay, a total of 375MW active powers are dropped at 3 seconds after system suffers disturbance, including 157.23MVar reactive power. The change curves of load bus voltage and system frequency are shown in Figure 5. Observing (b) of the Figure 5, under the new scheme, the system frequency can be restored to the safe and stable range in 15 seconds, and the maximum deviation of system frequency is only $0.1 \mathrm{~Hz}$. It is obviously better than the traditional UFLS scheme.

The new scheme drops less 117MW load than traditional UFLS scheme, but 40MVar more reactive load is removed. Observed in Figure 5 and (a) of the Figure 6, obviously, the voltage amplitude of bus 7 and bus 16 can be restored to higher values under the new scheme. Further analysis, if the system is in the removal of larger capacity of real and reactive power, the load bus voltage will be dropping rapidly, the traditional UFLS strategy will not be able to start at a predetermined time, the ability to system protection should be lost.

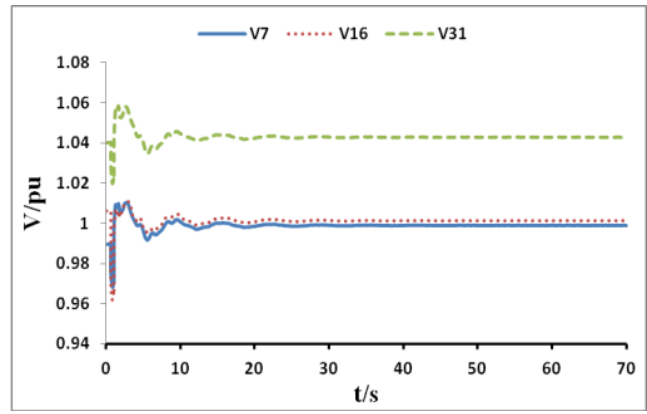

(a) Voltage change of the load bus

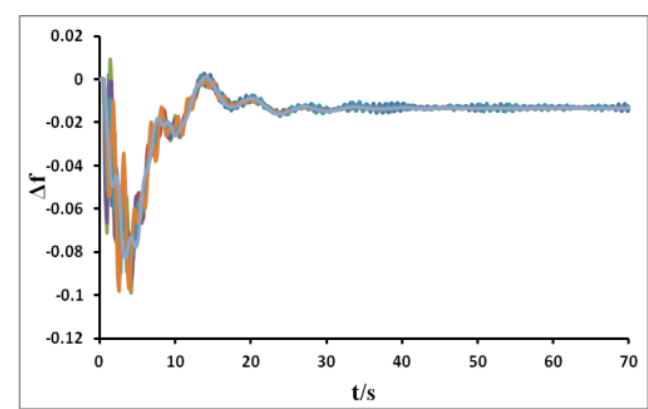

(b) The change of generator frequency

\section{Figure 5. the change curves of load bus voltage and generator frequency using new load shedding scheme}

\section{Conclusions}

5.1 The parameters, which influence generator rotor swing balance, are studied after the system suffers disturbance. It was put forward that the actual imbalance power in the system should be determined through two electric parameters, namely considering both the system frequency and the bus voltage simultaneously. And then, the accurate relationship equation for solving the imbalance power of disturbed power system is obtained. 
5.2 On the basis of voltage sensitivity analysis, a new emergency control strategy is advised that the position and the corresponding quantity of load shedding should be determined using voltage sensitivity. While real power imbalance of the system is recovered effectively, the local reactive power distribution should be also kept balanced. Load shedding should be carried out at weak points of the system, where the bus voltage and system frequency drop more or higher reactive power be needed. And this can improve the margin of system voltage stability, reducing the risk of a system breakdown.

5.3 The simulation examples show that the proposed new load shedding strategy has the stronger adaptability. The change of the system network topology can be considered, more or less of the load can be avoided. This scheme has more applied superiority and value.

\section{Acknowledgements}

Project was supported by the National Natural Science Foundation of China (Grant No.50837002) and The National Basic Research Program of China (973 Program) (2012CB215200)

\section{References}

[1] J. Zhang and T. B. Liu, "Review of frequency stability for islanded power system", Power System Protection and Control, vol. 39, no. 11, (2011), pp. 149-154.

[2] Z. Zhang, Z. Wang and P. Fang, "Emergency control strategy based on frequency and voltage stability", Power System Protection and Control, vol. 41, no. 3, (2013), pp. 149-155.

[3] X. Da, H. He and X. Chang, "An approach to design power system under frequency load shedding scheme taking coherent area and global optimization into account", Power System Technology, vol. 34, no. 6, (2010), pp. 106-112.

[4] B. Wang, W. Fang and X. Luo, "A fast algorithm of optimal generator and load-shedding for emergency control”, Power System Technology, vol. 35, no. 6, (2011), pp. 82-87.

[5] M. Qin and X. Yang, "Measures to reduce over shedding caused by under frequency load shedding project", Power System Technology, vol. 26, no. 3, (2002), pp. 83-86.

[6] S. Liu, C. Wang and J. An, "Study of under frequency load shedding scheme based on frequency static characteristics", Power System Protection and Control, vol. 3, no. 9, (2011), pp. 140-144.

[7] Q. Zhao and X. Wang, "A fast predictive algorithm for power system post disturbances steady frequency", Power System Protection and Control, vol. 39, no. 1, (2011), pp. 72-77.

[8] V. V. Terzija, "Adaptive under frequency load shedding based on the magnitude of the disturbance estimation”, IEEE Transactions on Power Systems, vol. 21, no. 3, (2006), pp. 1260-1266.

[9] D. L. H. Aik, "A general order system frequency response model incorporating load shedding: analytic modeling and applications[J]", IEEE Transactions on Power Systems, vol. 21, no. 2, (2006), pp. 709-717.

[10] X. Xiong, Y. Zhou and Z. Jiaqi, "Study of under frequency load shedding scheme based on load frequency characteristics", Proceedings of the CSEE, vol. 25, no. 19, (2005), pp. 48-51.

[11] H. You, V. Vital and Z. Yang. Self-healing in power systems: An approach using rate of frequency decline based load shedding", IEEE Transactions on Power Systems, vol. 18, no. 1, (2003), pp. 174-181.

[12] Z. Yuan and T. Xia, "An overview of setting method of under voltage load shedding", Power System Protection and Control, vol. 40, no. 15, (2012), pp. 136-14.

[13] X. Fu and X. Wang, "A new index of nodal static voltage stability and load shedding method", Power System Technology, vol. 30, no. 10, (2006), pp. 8-12, 17.

[14] M. S. Pasand and H. Seyedi, "New centralized adaptive under frequency load shedding algorithms", IEE Proceedings Generation, Transmission \& Distribution, vol. 3, no. 1, (2009), pp. 99-114.

[15] U. Rudez and R. Mihalic, "Analysis of under frequency load shedding using a frequency gradient", IEEE Transactions on Power Delivery, vol. 26, no. 4, (2011), pp. 565-575.

[16] H. Bin, Z. Liang and M. Shiying, "Undervoltage load shedding configuration based on PV curve", Power System Technology, vol. 32, no. 23, (2008), pp. 29-34.

[17] P. Kundur, "Power system stability and control”, New York: McGraw-Hill Inc., (1994), pp. 991-992.

[18] L. Xu, J. Lu and Y. Wang, "Research on nodal voltage stability index of power system", Power System Technology, vol. 34, no. 3, (2010), pp. 26-30. 


\section{Authors}

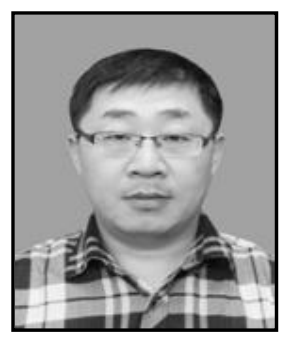

\section{Zhichao Zhang}

He received the M.S. degree in Electric Engineering from Shihezi University, China, in 2007. Now he is a teacher in College of Mechanical and Electronic Engineering, Shihezi University, China. Since 2008 he is a Ph.D student of North China Electric Power University. His current research interests include power system equipment protection, load shedding under emergency situations.

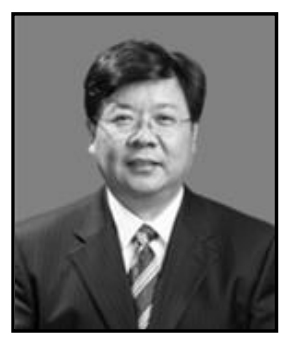

\section{Zengping Wang}

He received the B.S. and M.S. degree in Electric Engineering from North China Electric Power University, China, in 1985 and 1988, respectively. He received the Ph.D. degree from Harbin Institute of Technology, China, in 1997. He is a professor and the dean of the School of Electrical and Electronic Engineering at North China Electric Power University. His special fields of interest include power system equipment protection, fault analysis and wide-area protection.

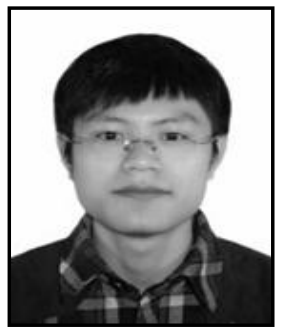

\section{Panyu Fang}

He received the B.S. degree in Electric Engineering from North China Electric Power University, China, in 2012. He is a second-year student of master's degree in North China Electric Power University in China, mainly majored in Power System Protection and Control. 